of the book is devoted to a descriptive, often case-history, treatment of individual problems and their attempted solution. Nearly half the text covers applications to enzymes and proteins, and it concludes with chapters on nucleic acids and on membranes. There are approximately 1,500 references (out of, we are told, a possible 6,000 on the subject) and a significant number of them are discussed at least briefly.

The style is refreshingly critical, with the inevitable result that every experienced reader will find points of disagreement with certain statements or with the emphasis. For example, I felt that the treatment of nuclear spin relaxation as a tool for studies of protein dynamics overemphasizes the methods used by Professor Jardetzky and his colleagues. The novice should be warned that nearly every topic in this field is subject to dispute but for the most part the authors do present both sides of complex issues, in references if not in their discussions. Unavoidably, certain areas are developing so rapidly that the text can provide only a useful indication of very recent advances: examples of such areas are studies of nucleic acids and of the conformation of small proteins by twodimensional NMR.

Jardetzky and Roberts have written the most thorough treatment of the subject now available, and the first such treatise to appear in some time. Their book will be extremely useful to both experienced NMR research workers and novices alike.

Alfred G. Redfield is Professor of Biochemistry and Physics at Brandeis University.

\title{
Tackling the bugaboos of solar flares
}

\section{Robert Rosner}

Solar Flare Magnetohydrodynamics. Edited by E.R. Priest. Pp.563. ISBN 0-677-05530-7. (Gordon \& Breach: 1982.) $\$ 89.50$.

SOLAR flares have long been an enigma. Along with such problems as how the solar corona is heated, and where the "missing" solar neutrinos are, the solar flare puzzle has been a persistent reminder to astrophysicists that the gap between observation and theory is still uncomfortably large whenever the data are of sufficient quality to rigorously test the details of theoretical models.

In the case of solar flares, the difficulties may be attributed both to the classic bugaboo of theoreticians, namely phenomena in which nonlinear effects dominate (in the present case, derived from the non-linear equations of magnetohydrodynamics); and to the virtual certainty that much of the crucial physics takes place on spatial scales which are not likely to be observable in the forseeable future (hence theory in this respect remains unconstrained by data). Given the complexity of the subject, it is welcome news that Solar Flare Magnetohydrodynamics does a commendable job of reviewing much of the theory relevant to present-day solar flare research.

The book is largely complementary to the recent NASA monograph on solar flares, Proceedings of the Skylab Workshop on Solar Flares edited by P.A. Sturrock (Colorado Associated University Press, 1980) and, if read in conjunction with this second tome, the student of solar flares will have in hand a full account of research prior to the recent flight of the Solar Maximum Mission (SMM) satellite. As such, the book should be of interest to all concerned with time-dependent (magneto-)hydrodynamics and plasma physics in astrophysics. The material is written at a fairly advanced level, but is not so esoteric as to be inaccessible to students.

With the exception of Z. Svestka's brief chapter which reviews observations (a topic covered far better in the NASA/ Skylab monograph), the book is true to its title and gives a systematic exposition of the physics of flaring plasmas, from equilibrium to possible instability, and along the way treats the associated microphysics leading to explosive heating and particle acceleration. Chapters are written by active, prominent researchers in the field, and generally convey a good sense of the current status of work in this area. Particularly noteworthy and complete are the discussions of current sheet dynamics by $E$. Priest, time-dependent hydrodynamics (I. Craig), evolution of post-flare plasma (G. Pneuman) and flare particle acceleration processes (J. Heyvaerts).

With the exception of $\mathrm{J}$. Birn and $\mathrm{K}$. Schindler's discussion of magnetic field equilibria, the chapters are well-integrated; this exceptional case, though competent in content, has a rather formal style, with virtually no attempt to mesh theory with observation.

A pleasant surprise is Priest's superb overview in the introduction, in which a precis of the entire book is given; this chapter not only ties the various contributions together, but also gives a nice perspective of the solar flare problem as a whole. The overall result is a monograph of great value; it is a must.for solar physicists, and for all interested in sudden magnetic energy release and its consequences in astrophysics.

Robert Rosner is an Assistant Professor of Astronomy at Harvard University.
Energy and life

\section{Robert M. Macnab}

Biological Energy Transduction: The Uroboros. By Ronald F. Fox. Pp.279. ISBN 0-471-09026-3. (Wiley: 1982.) $£ 24.50, \$ 43.80$.

THE dual purpose of this book is given in its preface: first, it is intended to be an introduction, for physical scientists, to advances that have taken place in molecular biology in the past few decades; second, a description of biology from the viewpoint of energetics, suitable for senior undergraduates or graduates. These would seem to be rather distinct aims, and it is unclear why they should be pursued in a single volume - or, for that matter, why physical scientists require a special exposition of molecular biology.

An emphasis on energetic considerations in biology and their use as a unifying concept is what the title of the book leads one to expect, and, in general, the author has been successful in presenting this point of view. His style is clear and entertaining, and the major topics of interest in bioenergetics (e.g. control of metabolic pathways, storage of energy across membranes, chemi-osmosis, energetics of macromolecular synthesis) are treated well, provided one does not examine too closely the factual details presented.

In this latter regard, the book is flawed. Sometimes the error (e.g. that the bacterial flagellum is a triple helix) is incidental to the argument, but it is irritating nonetheless and one wonders how it came about. In other instances, the errors are more serious. I will cite two examples. First, on p.203, an erroneously high value for the cytoplasmic $p \mathrm{H}$ of bacteria is given, and then used in subsequent bioenergetic calculations. The author comments that

Many microbiologists and biochemists will be surprised to read that for bacteria grown in a medium of $p \mathrm{H} 7$ the internal $p \mathrm{H}$ is as high as 9 .

Indeed they will — did he ever ask them? (Typical experimental values are around 7.5.) Second, the choice of standard state for $\mathrm{H}_{2} \mathrm{O}$ in conventional tabulations of free energy changes, redox potentials and so on, is incorrectly believed by the author (p.62) to be $1 \mathrm{M}$; based on this belief, he proceeds to make adjustments to $55.6 \mathrm{M}$, which of course was the standard state chosen in the original tabulations. A variety of conclusions regarding the energetics of electron transport and oxidative phosphorylation are then based on these adjusted values, and the rest of the scientific community is taken to task (p.211) for failing to make these "corrections". It is unfortunate that the author apparently did not discuss these questions with physical chemists or biochemists before codifying his viewpoint in a textbook.

The final part of the book is devoted to a 